\title{
Independent control of exciton and biexciton energies in single quantum dots via electroelastic fields
}

\author{
Rinaldo Trotta, ${ }^{1,2, *}$ Eugenio Zallo, ${ }^{2}$ Elisabeth Magerl, ${ }^{2,3}$ Oliver G. Schmidt, ${ }^{2,4}$ and Armando Rastelli ${ }^{1,2}$ \\ ${ }^{1}$ Institute of Semiconductor and Solid State Physics, Johannes Kepler University Linz, Altenbergerstraße 69, A-4040 Linz, Austria \\ ${ }^{2}$ Institute for Integrative Nanosciences, IFW Dresden, Helmholtzstraße 20, D-01069 Dresden, Germany \\ ${ }^{3}$ Photonics Institute, Vienna University of Technology, Gusshausstrasse 25, 1040 Vienna, Austria \\ ${ }^{4}$ Center for Advancing Electronics Dresden, Technische Universität Dresden, Georg-Schumann-Str. 11, 01187 Dresden, Germany
}

(Received 10 August 2013; published 15 October 2013)

\begin{abstract}
We investigate the effect of large in-plane strain and vertical electric fields on the binding energies of excitonic complexes confined in single InGaAs/GaAs quantum dots (QDs) and we find that the two independently tunable perturbations modify the interaction energies among electrons and holes in a different manner. By taking advantage of this difference, we frequency-lock the QD fundamental excitation (the neutral exciton) at a predefined value, while the biexciton transition is actively tuned from a binding to an antibinding configuration. Our electrically controlled dual-knob device demonstrates unprecedented control over the electronic properties of the few-particle states in a QD and may be applied to create novel energy-tunable sources of entangled photons using the time-reordering or the time-bin scheme.
\end{abstract}

DOI: $10.1103 /$ PhysRevB.88.155312

PACS number(s): 78.67.Hc, 85.35.Be, 81.07.Ta

Semiconductor quantum dots (QDs) are considered promising building blocks of future solid-state quantum communication, ${ }^{1-3}$ mainly due to their capability to generate nonclassical light ${ }^{4-6}$ and to act as convenient hosts of spin qubits. ${ }^{7,8}$ These potential applications are built around fewparticle states in a QD, whose electronic properties are governed by the interplay between quantum confinement, direct Couloumb interactions among charged carriers, and exchange and correlation effects. ${ }^{9,10}$ Despite the remarkable progress achieved in the control and manipulation of semiconductor materials at the nanoscale, ${ }^{11,12}$ these quantum interactions turned out to be so sensitive to the QD structural details that, unlike their natural counterpart, each "artificial atom" has unique electronic properties. A prominent example is represented by the few-particle-state recombinations of excitonic complexes confined in a $\mathrm{QD}$, such as the biexciton $(X X)$ and the negatively and the positively charged trions $\left(X^{+}\right.$and $\left.X^{-}\right)$. Their relative binding energy, i.e., their energetic position with respect to the QD fundamental excitation (the neutral exciton, $X$ ), is dramatically affected by the QD size, shape, strain, and composition. ${ }^{10,13,14}$ This represents a major obstacle for the implementation of quantum networks ${ }^{15}$ based on flying QD-based qubits, where quantum emitters with identical emission properties should be readily available. Reaching the latter goal, in particular, is the main driving force behind current efforts to engineer the emission properties of semiconductor QDs via external perturbations. ${ }^{16-24}$ Magnetic-- ${ }^{16}$ electric-, ${ }^{17-20}$ and piezoelectric-induced strain ${ }^{21-24}$ fields have proved to modify emission energies, charge states, and energylevel splittings in a precise manner, and proof of principle experiments aiming at interfacing distant QD-based qubits have been performed. ${ }^{25,26}$ However, the departure from this pioneering phase requires a higher degree of control over the QD emission properties, which would allow advanced quantum optics experiments currently inaccessible to QDs, such as entanglement swapping between distant qubits, to be performed. ${ }^{27}$

In this work, we show unprecedented control over the QD emission properties via an electrically controlled device, which combines two independent "tuning knobs": strain and electric fields. We first investigate in detail the separate effects produced by the two external perturbations on the relative binding energy of the excitonic complexes confined in the same QD. We then demonstrate independent control of the emission energies of two excitonic species, and in particular of the $X$ and $X X$. Compared to recent reports, ${ }^{17,18,24}$ which have shown only the tunability of the relative emission energy (with $X$ and $X X$ both shifting under the application of an external field), the relative binding energy of $X X$ is continuously tuned, while the fundamental excitation of a QD - the exciton, $X$-is frequency-locked at a predefined value. This result shows that the combination of independent external fields does not simply extend the tunability of the QD emission properties but allows for a reconfiguration of the interaction energies among carriers in a manner inaccessible with a single perturbation. Our approach lends itself to create novel energy-tunable sources of entangled photon pairs using the time-reordering or the time-bin scheme, ${ }^{6,28}$ a fundamental ingredient for advanced quantum optics experiments with QDs.

A sketch of the dual-knob device employed in this work is shown in Fig. 1(a). Molecular beam epitaxy was used to grow low-density InGaAs QDs in the intrinsic region of an $n-i$ - $p$ diode. The diode contains $\mathrm{Al}_{0.4} \mathrm{Ga}_{0.6} \mathrm{As}$ barriers surrounding a 10-nm-thick GaAs quantum well, which, in turn, hosts the QDs. The presence of the $\mathrm{Al}_{0.4} \mathrm{Ga}_{0.6} \mathrm{As}$ barriers allows large electric fields $\left(F_{d}\right.$ up to $\sim 250 \mathrm{kV} / \mathrm{cm}$ ) to be applied before electrons and holes tunnel out of the QD. ${ }^{20}$ This results in a broad range control of the QD emission lines by tuning the voltage applied to the diode, $V_{d}$ [see Fig. 1(d)]. The device was grown on an $\mathrm{Al}_{0.75} \mathrm{Ga}_{0.25} \mathrm{As}$ sacrificial layer, which we use for substrate removal and for the subsequent transfer (via gold thermocompression bonding) of the $\sim 430$-nm-thick membrane-diodes onto single-crystal $\left[\mathrm{Pb}\left(\mathrm{Mg}_{1 / 3} \mathrm{Nb}_{2 / 3}\right) \mathrm{O}_{3}\right]_{0.72}\left[\mathrm{PbTiO}_{3}\right]_{0.28}$ (PMN-PT) substrates. ${ }^{29}$ The PMN-PT equips the device with an additional tuning knob: strain. The in-plane strain $\varepsilon_{\|}$in the QD layer can be varied by an amount as large as $\Delta \varepsilon_{\|} \sim 0.4 \%$ by applying an electric field $F_{p}$ (via $V_{p}$ ) across the PMN-PT, and the QD optical properties can be tuned in a well-defined manner ${ }^{29}$ [see Fig. 1(e)]. We refer the reader to Ref. 29 for further details about sample growth and device fabrication. Figure 1(b) 
(a)

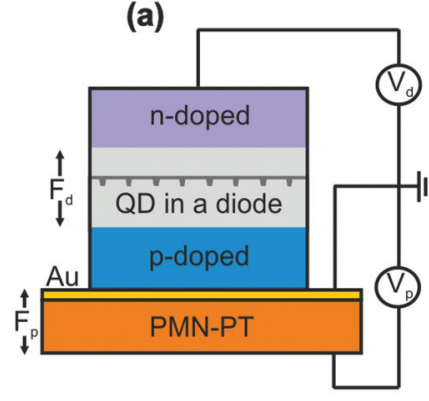

(b)
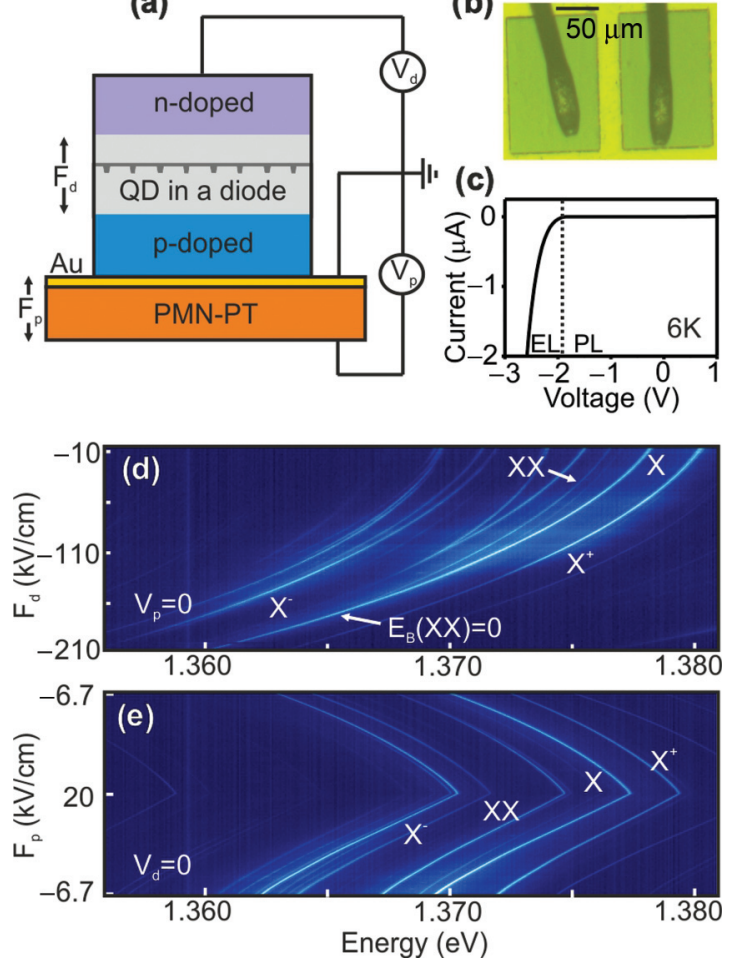

FIG. 1. (Color online) (a) Sketch of the dual-knob device employed in this work, where membrane diodes are integrated on top of piezoelectric actuators (PMN-PT). $V_{d}\left(V_{p}\right)$ controls the electric field (strain field) across the QDs. (b) Optical microscopy image of two of the devices used in this work. The green (yellow) areas are the membrane-diodes (gold-coated PMN-PT). A 25- $\mu$ m-thick Al wire was used for contacting the $n$-doped layer of the membrane. (c) Low-temperature $(T=6 \mathrm{~K})$ current $(I)$ - voltage $\left(V_{d}\right)$ characteristic of one of the devices. (d) Color-coded $\mu$-PL map of a single QD embedded in the device as a function of the electric field across the QD $\left(F_{d}\right)$ and with $V_{p}=0$. A logarithmic scale is used for the intensity. Several emission lines from the same QD can be observed: exciton $(X)$, biexciton $(X X)$, negatively and positively charged trions $\left(X^{-}\right.$and $\left.X^{+}\right)$. (e) Color-coded $\mu$-PL map of the same QD as in (d) as a function of the electric field across the PMN-PT $\left(F_{p}\right)$ and with $V_{d}=0$. Positive (negative) electric fields $F_{p}$ correspond to in-plane compressive (tensile) stress.

shows an optical microscope image of two of the devices used in this work, where the membrane-diodes integrated on the PMN-PT can be observed. A typical current $(I)-$ voltage $\left(V_{d}\right)$ characteristic of the device [see Fig. 1(c)] shows an almost perfect diodelike behavior, with no sizeable leakage current for reverse bias. It is important to note that the bias applied to the diode has a dual role here: It can be used in synergy with strain to modify the emission properties of the QD (reverse bias, $V_{d}>-1.9 \mathrm{~V}$ ) or can be taken up for injecting carriers electrically (forward bias, $V_{d}<-1.9 \mathrm{~V}$ ), thus exciting electroluminescence. In this work, the diodes are always operated in reverse bias.

Microphotoluminescence ( $\mu$-PL) spectroscopy was used to address the optical properties of single QDs. The measurements were performed at low temperature (typically 4-10 K) in a helium flow cryostat. The QDs were excited with an 850-nm continuous-wave laser, and the PL signal was collected with a spectrometer featuring $\sim 30-\mu \mathrm{eV}$ resolution at $900 \mathrm{~nm}$ and equipped with a liquid nitrogen-cooled Si charge-coupled device camera. Power and polarization resolved measurements (performed with a rotating $\lambda / 2$ wave plate and a fixed linear polarizer placed in front of the spectrometer) were used to identify the exciton $(X)$ and the biexciton $(X X)$ transitions. The negative $\left(X^{-}\right)$and positive $\left(X^{+}\right)$trions were recognized by the absence of fine structure and investigating their energy shifts under application of stress (see the following and Ref. 24). The assignment was also confirmed by embedding the same type of QDs in charge-tunable devices $[n-i$-Schottky diodes, in the same configuration displayed in Fig. 1(a)], where the appearance of the different complexes with increasing $V_{d}$ identifies unambiguously their origin. ${ }^{8}$

Figures 1(d) and 1(e) show color-coded $\mu$-PL maps of single QDs as a function of $F_{d}$ and $F_{p}$, respectively. The two fields allow the QD emission lines to be shifted in a very broad spectral range, which can be as large as $30 \mathrm{meV}$ when the device is operated in "additive mode", i.e., when the two fields are used to shift the emission in the same direction. ${ }^{21}$ Furthermore, Fig. 1(d) shows that the energy separation between $X$ and $X X$ emission lines, which we refer to as the relative biexciton binding energy and defined as $E_{B}(X X)=$ $E_{X}-E_{X X}$ (where $E_{X, X X}$ are the emission energies), can be widely tuned, so that $X X$ and $X$ can be brought into energy coincidence with the only application of $F_{d}$ even when their initial $E_{B}$ is as large as $2 \mathrm{meV}$. In combination with strain, the device features $\sim 3-\mathrm{meV}$ tunability of $E_{B}$. Since this value is much larger than the average $\left|E_{B}(X X)\right|$ found in our QDs at $V_{d}=V_{p}=0$ (1.43 meV, averaged over 35 QDs), the additive mode operation of the device allows $X-X X$ color coincidence to be achieved in almost all the QDs in the ensemble. This result is relevant for the generation of entangled phonon pairs using the time-reordering scheme. ${ }^{28}$ Another interesting feature clearly visible in Figs. 1(d) and 1(e) is that $X, X X$, $X^{+}$, and $X^{-}$shift at different rates with the two fields. These differences are of major interest for this work, as discussed in the following.

Figure 2(a) shows four $\mu$-PL spectra of different QDs embedded in a device at $V_{d}=V_{p}=0$. A few common features of InGaAs QDs emerge from these spectra: ${ }^{13,14,30}$ (i) the relative biexciton binding energy can be either positive or negative and does not show any correlation with the energy of the exciton transition $\left(E_{X}\right)$; (ii) the ordering of the trion transitions appears to be fixed, i.e., the positive (negative) trion is always on the high- (low-) energy side of the exciton. Furthermore, the absolute value of the relative binding energy of the negative trion is usually larger than that of the positive trion and of the order of a few millielectron volts. (ii) can be explained considering the magnitude of the direct Coulomb integrals $\left(J^{i j}\right)$ between electrons $(i, j=e)$ and holes $(i, j=h)$ confined in this type of QDs, which feature a nominal height of $2 \mathrm{~nm}$. In fact, if correlation and exchange effects can be neglected, the relative binding energies of the different excitonic complexes can be written as $E_{B}\left(X^{-}\right)=-J^{e e}+\left|J^{e h}\right|, E_{B}\left(X^{+}\right)=-J^{h h}+\left|J^{e h}\right|$, and $E_{B}(X X)=-J^{e e}-J^{h h}+2\left|J^{e h}\right|$. Since in $\operatorname{In}(\mathrm{Ga}) \mathrm{As} / \mathrm{GaAs}$ QDs, the hole wave function is much more localized than the electron wave function (due to the larger effective mass) and the centers of mass of the two carriers are close, the following 

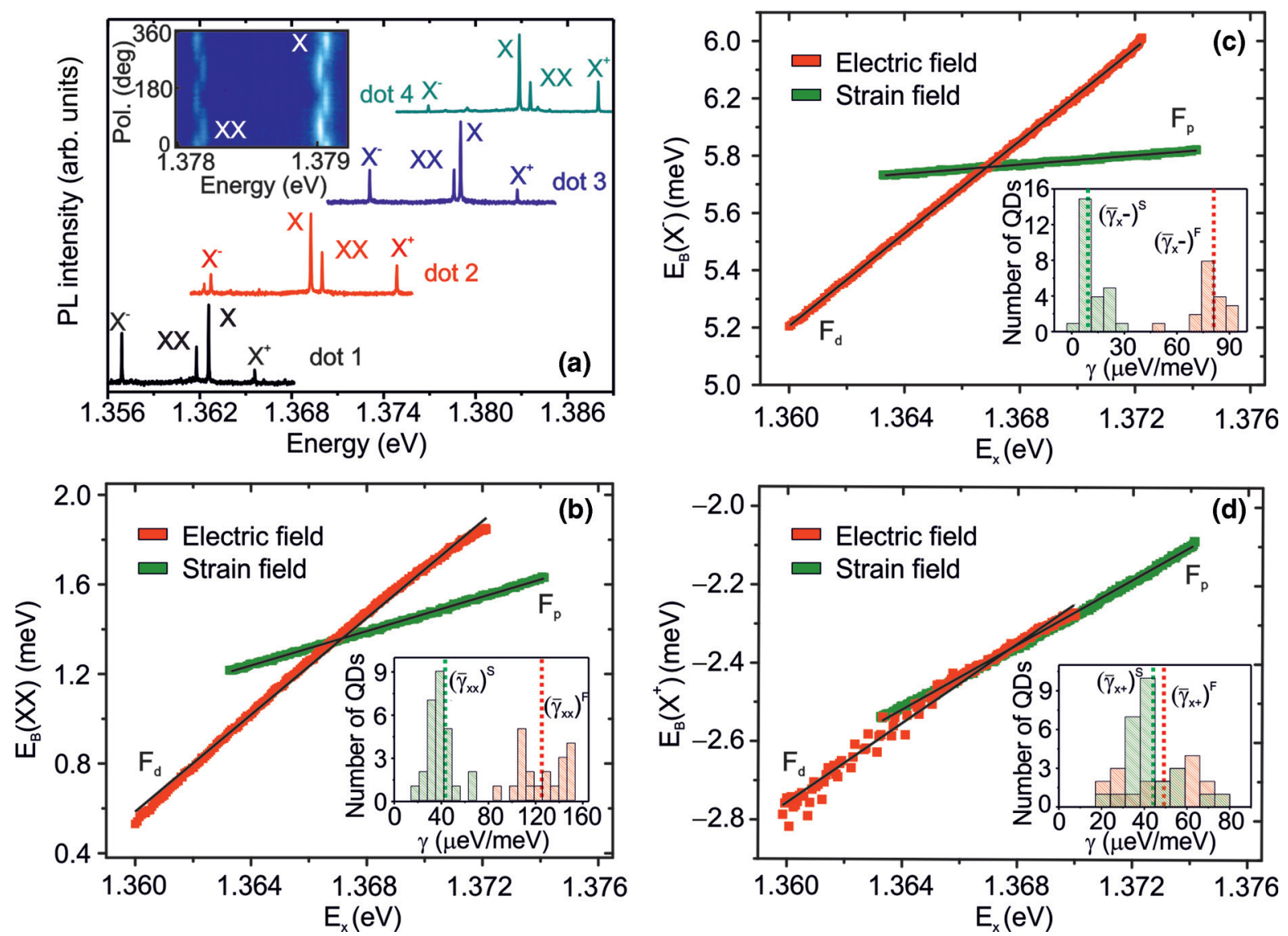

FIG. 2. (Color online) (a) $\mu$-PL spectra of several QDs at $V_{p}=V_{d}=0$. In the inset, a polarization resolved $\mu$-PL map of a single QD is also displayed. (b)-(d) Relative binding energies of the biexciton (b) and negative (c) and positive (d) trions as a function of the energy of the exciton transition for a single QD. Red (green) squares refer to the data obtained when $F_{d}\left(F_{p}\right)$ is varied at $F_{p}=0\left(F_{d}=0\right)$. Black lines are linear fits to the experimental data. In the inset of each panel, a histogram of the field-induced changes of the relative binding energies normalized by the change in energy of the exciton transition ( $\gamma$; see main text) is also reported for all the measured QDs. The dashed lines indicate the averaged values.

relation holds: $J^{e e}<\left|J^{e h}\right|<J^{h h}$ (see Refs. 10 and 31 for more details). This implies that the negative (positive) trion is always at the low- (high-) energy side of the exciton in this kind of QDs, as observed experimentally. It is also worth noting that the magnitudes of $E_{B}\left(X^{-}\right)$and $E_{B}\left(X^{+}\right)$match atomistic calculations of InGaAs QDs with similar height. ${ }^{31}$ For the biexciton, correlation and exchange interactions have to be taken into account for reproducing the binding and antibinding nature of this complex. ${ }^{10,31}$ However, the simple model discussed above is still useful for investigating how the relative binding energies of the excitonic complexes react when different external fields are applied.

When the voltages are varied, the relative binding energies of all the species show a quadratic (linear) change with the magnitude of the electric field (strain), while they always display a linear behavior when they are plotted against the exciton energy, as shown in Figs. 2(b)-2(d) for a specific QD. This allows us to define "rates" at which the relative binding energies change with the exciton emission energy $\left(\gamma=\Delta E_{B} / \Delta E_{X}\right)$ for the two fields. Different QDs embedded in different devices were studied, and the resulting analysis is displayed in the form of histograms in the insets of Figs. 2(b)-2(d). It is quite clear that strain $(S)$ and electric $(F)$ fields affect the relative binding energies of the excitonic complexes in a different manner, being $(\bar{\gamma})^{F}>(\bar{\gamma})^{S},\left(\bar{\gamma}_{X X}\right)^{S} \sim\left(\bar{\gamma}_{X^{+}}\right)^{S}>\left(\bar{\gamma}_{X^{-}}\right)^{S}$ and $\left(\bar{\gamma}_{X X}\right)^{F}>\left(\bar{\gamma}_{X^{-}}\right)^{F}>\left(\bar{\gamma}_{X^{+}}\right)^{F}$ (where $\bar{\gamma}$ is the average $\gamma$; see insets of Fig. 2). This suggests that there is a fielddependent modification of the interaction energies among electrons and holes confined into the same QDs. If we consider only direct Coulomb interactions, the variation of the relative binding energies are $\Delta E_{B}\left(X^{-}\right)=-\Delta J^{e e}+$ $\Delta\left|J^{e h}\right|, \Delta E_{B}\left(X^{+}\right)=-\Delta J^{h h}+\Delta\left|J^{e h}\right|$ and $\Delta E_{B}(X X)=$ $\Delta E_{B}\left(X^{+}\right)+\Delta E_{B}\left(X^{-}\right)$. The last equation is reproduced qualitatively by the experimental data, where $\bar{\gamma}_{X X} \sim \bar{\gamma}_{X^{-}}+$ $\bar{\gamma}_{X^{+}}$. This agreement supports the assumptions of our simple model and allows us to focus the discussion on the trion transitions only. In this context, it is interesting to evaluate the following quantity:

$$
\delta=\Delta E_{B}\left(X^{-}\right)-\Delta E_{B}\left(X^{+}\right)=\Delta J^{h h}-\Delta J^{e e} .
$$

In fact, we find that $\delta$ has different sign when strain or electric fields are applied to the same QD, so as to increase or decrease $E_{X}$. In particular, under electric fields $\left(\Delta J^{e e}\right)^{F}<\left(\Delta J^{h h}\right)^{F}$ when the $X$ emission energy is 


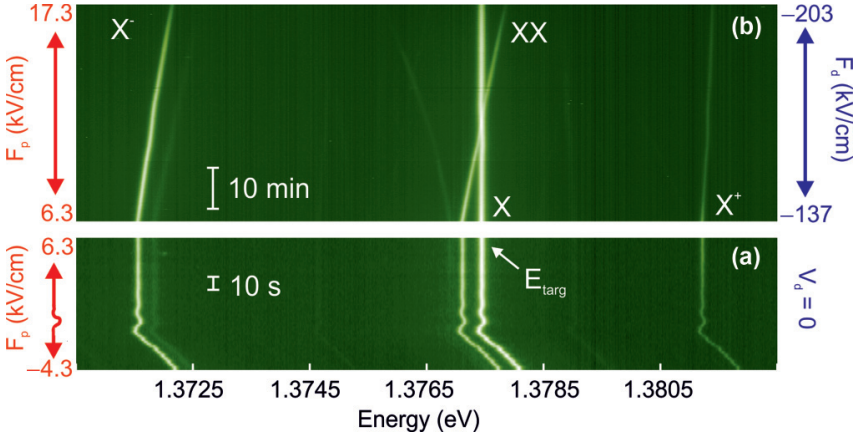

FIG. 3. (Color online) Independent control of $X$ and $X X$ emission energies for a single QD. The time evolution of the $\mu$-PL signal of a QD is shown as the applied fields are varied. (a) Color-coded $\mu$-PL map of a single QD whose exciton transition is first tuned to the target energy of $E_{\text {targ }}=1.3774 \mathrm{eV}$ and then locked at this value via a computer-controlled active feedback on $F_{p}$. During the experiment, $V_{d}=0$. The abscissa indicates the energy of the emitted photons. Because of fast feedback parameters, some oscillations can be noticed in the time-trace before $E_{x}$ finally reaches a stable value. (b) Color-coded $\mu$-PL map of the same QD in (a) when the magnitude of $F_{d}$ is ramped up, while the exciton transition is locked at $E_{\text {targ }}$ via $F_{p}$, as explained in the main text.

blue-shifted. The opposite situation is instead found with strain, where $\left(\Delta J^{e e}\right)^{S}>\left(\Delta J^{h h}\right)^{S}$. As explained in Ref. 24, this is because an in-plane compressive biaxial strain increases the confinement potential of electrons-and consequently their Coulomb repulsion-while it slightly reduces the hole repulsion. The electron-hole attraction also increases as a consequence of an improved electron-hole overlap. This picture is confirmed by Figs. 2(b)-2(d), which indicates that the strain-induced changes in $J^{e h}$ are almost counterbalanced by the changes in $J^{e e}$. This is not the case for electric field. Assuming that $J^{e h}$ varies little with varying electric field, ${ }^{32}$ the large value of $\left(\gamma_{X^{-}}\right)^{F}$, indicates that $\Delta J^{e e}$ is negative for increasing $E_{X}$, which we attribute to the fact that an increasing electric field (decreasing exciton energy) brings electrons closer to each other. The different inequalities we find for electric and strain fields can thus be explained in the following manner: while both fields have a larger effect on the absolute value of the Coulomb integrals between electrons than between holes, the change in electron-electron repulsion under strain and electric field have opposite signs.

The data reported so far clearly show that strain and electric fields have a different influence on the relative position and spatial extent of the carrier wave functions. We now illustrate how the different physical effects produced by the two external perturbations can be exploited to actively reconfigure the electronic properties of a QD so that the absolute emission energies of two different excitonic species are tuned independently of each other. To do so, we operate our dual-knob device in "subtractive mode", i.e., we let the two external fields work "against" each other, as illustrated in the experiment presented in Fig. 3 and discussed in the following. We first use strain (i.e., the computer-controlled voltage $V_{p}$ applied to the PMN-PT) to shift and frequency-lock, with microelectron volt precision, ${ }^{29}$ the $X$ transition to a user-defined target energy
( $\left.E_{\text {targ }}\right)$ [see Fig. 3(a)]. This is achieved by comparing the actual exciton energy $E_{X}$ (obtained via a Lorentzian fitting of the exciton line) with $E_{\text {targ }}$ and adjusting automatically $V_{p}$ in order to minimize $E_{X}-E_{\operatorname{targ}}$. We then change linearly $V_{d}$ to increase the magnitude of $F_{d}$, while the exciton transition is kept fixed at $E_{\text {targ }}$ via $V_{p}$ [see Fig. 3(b)]. In absence of the feedback, all the QD emission lines would redshift due to the quantum confined Stark effect. However, as the $X$ shift is actively compensated by increasingly compressive strain, we only change the spectral position of the other excitonic complexes. Considering that the $(\gamma)^{S}\left((\gamma)^{F}\right)$ defined above does not depend appreciably on the value of $F_{d}$ $\left(F_{p}\right)$, we observe that under the simultaneous application of strain and electric field the trions and biexciton energies shift at a rate $(d E / d t)_{F-S}=\left[(\gamma)^{F}-(\gamma)^{S}\right] \cdot\left(d E_{X} / d t\right)^{S}$, being $\left(d E_{X} / d t\right)^{S}=-\left(d E_{X} / d t\right)^{F}(\sim 3 \mu \mathrm{eV} / \mathrm{s}$ in the experiment $)$. The different values of $(\gamma)^{F}$ and $(\gamma)^{S}$, which are a direct consequence of the different effects strain and electric field have on the electron and hole wave functions, guarantee the tunability of the emission energy of trions and biexciton at a fixed exciton energy. Remarkably, in the QD of Fig. 3 the biexciton changes gradually from a binding to an antibinding configuration for a fixed and predefined exciton energy $\left(E_{\mathrm{targ}}\right)$. Thus, the relative binding energies of excitonic complexes are modified without affecting the energy of the fundamental QD excitation, i.e., the neutral exciton. This highlights that the combination of independent external fields allows for active reconfiguration of the quantum interactions among charge carriers confined in QD in a manner inaccessible with single perturbations used alone. It is also worth noting that the stabilization scheme employed in Fig. 3 allows $X-X X$ color coincidence to be achieved at different values of the $X$ energy (by simply choosing a different $E_{\text {targ }}$ ). This would allow the development of energy-tunable sources of entangled photons via the recently proposed and not yet experimentally demonstrated time reordering-scheme, ${ }^{28}$ and it would pave the way towards entanglement swapping between distant QD-based qubits. If it turns out that the entanglement fidelity achievable via the time-reordering is not sufficient to reach this goal, ${ }^{33,34}$ the same task can be performed combining the experimentally demonstrated time-bin scheme ${ }^{6}$ with the remarkable control over the relative biexciton binding energy demonstrated in Fig. 2.

In summary, we have investigated the combined effect of strain and electric fields, provided by strain-tunable optoelectronic devices, on the optical properties of single semiconductor QDs. We find that the two fields modify the interaction energies among electrons and holes in a different manner. These differences allow for a reshaping of the QD electronic properties. In particular, we demonstrate that when the two external perturbations work one "against" the other, the biexciton binding energy can be modified from a binding to an antibinding configuration without affecting the energy of the exciton transition. On the one hand, the unprecedented control over the biexciton binding energy can be exploited for the fabrication of energy-tunable sources of entangled photons via the time-reordering or the time-bin scheme., ${ }^{6,28}$ On the other hand, our concept of combining different external perturbations to achieve independent control of different QD parameters can be exploited in the emerging field of quantum 
communication, where quantum emitters with reconfigurable emission properties may be a fundamental requirement.

\section{ACKNOWLEDGMENTS}

We thank P. Atkinson, S. Kumar, and J. D. Plumhof for fruitful discussions; J. R. Schröter, B. Eichler, R. Engelhardt, and D. Grimm for technical support; and K. Dörr and A. Herklotz for help with the piezoelectric actuators. The work was supported financially by Bundesministerium für Bildung und Forschung (BMBF) project QuaHL-Rep (Contract No. 01BQ1032) and by European Union Seventh Framework Programme 209 under Grant Agreement No. 601126210 (HANAS).
*Corresponding author: rinaldo.trotta@jku.at

${ }^{1}$ A. J. Shields, Nat. Photonics 1, 215 (2007).

${ }^{2}$ K. De Greve, L. Yu, P. L. McMahon, J. S. Pelc, C. M. Natarajan, N. Young Kim, E. Abe, S. Maier, C. Schneider, M. Kamp, S. Höfling, R. H. Hadfield, A. Forchel, M. M. Fejer, and Y. Yamamoto, Nature (London) 491, 421 (2012).

${ }^{3}$ W. B. Gao, P. Fallahi, E. Togan, J. Miguel-Sanchez, and A. Imamoglu, Nature (London) 491, 426 (2012).

${ }^{4}$ P. Michler, A. Kiraz, C. Becher, W. V. Schoenfeld, P. M. Petroff, L. Zhang, E. Hu, and A. Imamoglu, Science 290, 2282 (2000).

${ }^{5}$ C. L. Salter, R. M. Stevenson, I. Farrer, C. A. Nicoll, D. A. Ritchie, and A. J. Shields, Nature (London) 465, 594 (2010).

${ }^{6}$ H. Jayakumar, A. Predojević, T. Kauten, T. Huber, G. S. Solomon, and G. Weihs, arXiv:1305.2081.

${ }^{7}$ K. De Greve, P. L. McMahon, D. Press, T. D. Ladd, D. Bisping, C. Schneider, M. Kamp, L. Worschech, S. Höfling, A. Forchel, and Y. Yamamoto, Nat. Phys. 7, 872 (2011).

${ }^{8}$ B. D. Gerardot, D. Brunner, P. A. Dalgarno, P. Öhberg, S. Seidl, M. Kroner, K. Karrai, N. G. Stoltz, P. M. Petroff, and R. J. Warburton, Nature (London) 451, 441 (2008).

${ }^{9}$ M. Ediger, G. Bester, A. Badolato, P. M. Petroff, K. Karrai, A. Zunger, and R. J. Warburton, Nat. Phys. 3, 774 (2007).

${ }^{10}$ A. Schliwa, M. Winkelnkemper, and D. Bimberg, Phys. Rev. B 79, 075443 (2009).

${ }^{11}$ G. Juska, V. Dimastrodonato, L. O. Mereni, A. Gocalinska, and E. Pelucchi, Nat. Photonics 7, 527 (2013).

${ }^{12}$ R. Trotta, A. Polimeni, F. Martelli, G. Pettinari, M. Capizzi, L. Felisari, S. Rubini, M. Francardi, A. Gerardino, P. C. M. Christianen, and J. C. Maan, Adv. Mater. 23, 2706 (2011).

${ }^{13}$ S. Rodt, R. Heitz, A. Schliwa, R. L. Sellin, F. Guffarth, and D. Bimberg, Phys. Rev. B 68, 035331 (2003).

${ }^{14}$ R. J. Young, R. M. Stevenson, A. J. Shields, P. Atkinson, K. Cooper, D. A. Ritchie, K. M. Groom, A. I. Tartakovskii, and M. S. Skolnick, Phys. Rev. B 72, 113305 (2005).

${ }^{15}$ H. J. Kimble, Nature (London) 453, 1023 (2008).

${ }^{16}$ R. M. Stevenson, R. J. Young, P. See, D. G. Gevaux, K. Cooper, P. Atkinson, I. Farrer, D. A. Ritchie, and A. J. Shields, Phys. Rev. B 73, 033306 (2006).
${ }^{17}$ M. E. Reimer, M. P. van Kouwen, A. W. Hidma, M. H. M. van Weert, E. P. A. M. Bakkers, L. P. Kouwenhoven, and V. Zwiller, Nano Lett. 11, 645 (2011).

${ }^{18}$ M. Kaniber, M. F. Huck, K. Müller, E. C. Clark, F. Troiani, M. Bichler, H. J. Krenner, and J. J. Finley, Nanotechnology 22, 325202 (2011).

${ }^{19}$ M. M. Vogel, S. M. Ulrich, R. Hafenbrak, P. Michler, L. Wang, A. Rastelli, and O. G. Schmidt, Appl. Phys. Lett. 91, 051904 (2007).

${ }^{20}$ A. J. Bennett, M. A. Pooley, R. M. Stevenson, M. B. Ward, R. B. Patel, A. Boyer de la Giroday, N. Sköld, I. Farrer, C. A. Nicoll, D. A. Ritchie, and A. J. Shields, Nat. Phys. 6, 947 (2010).

${ }^{21}$ R. Trotta, E. Zallo, C. Ortix, P. Atkinson, J. D. Plumhof, J. van den Brink, A. Rastelli, and O. G. Schmidt, Phys. Rev. Lett. 109, 147401 (2012).

${ }^{22}$ C. E. Kuklewicz, R. N. E. Malein, P. M. Petroff, and B. D. Gerardot, Nano Lett. 12, 3761 (2012).

${ }^{23}$ S. Kumar, R. Trotta, E. Zallo, J. D. Plumhof, P. Atkinson, A. Rastelli, and O. G. Schmidt, Appl. Phys. Lett. 99, 161118 (2011).

${ }^{24}$ F. Ding, R. Singh, J. D. Plumhof, T. Zander, V. Křápek, Y. H. Chen, M. Benyoucef, V. Zwiller, K. Dörr, G. Bester, A. Rastelli, and O. G. Schmidt, Phys. Rev. Lett. 104, 067405 (2010).

${ }^{25}$ E. B. Flagg, A. Muller, S. V. Polyakov, A. Ling, A. Migdall, and G. S. Solomon, Phys. Rev. Lett. 104, 137401 (2010).

${ }^{26}$ R. B. Patel, A. J. Bennett, I. Farrer, C. A. Nicoll, D. A. Ritchie, and A. J. Shields, Nat. Photonics 4, 632 (2010).

${ }^{27}$ H. de Riedmatten, I. Marcikic, J. A. W. van Houwelingen, W. Tittel, H. Zbinden, and N. Gisin, Phys. Rev. A 71, 050302(R) (2005).

${ }^{28}$ J. E. Avron, G. Bisker, D. Gershoni, N. H. Lindner, E. A. Meirom, and R. J. Warburton, Phys. Rev. Lett. 100, 120501 (2008).

${ }^{29}$ R. Trotta, P. Atkinson, J. D. Plumhof, E. Zallo, R. O. Rezaev, S. Kumar, S. Baunack, J. R. Schröter, A. Rastelli, and O. G. Schmidt, Adv. Mater. 24, 2668 (2012).

${ }^{30}$ C. Jarlov, P. Gallo, M. Calic, B. Dwir, A. Rudra, and E. Kapon, Appl. Phys. Lett. 101, 191101 (2012).

${ }^{31}$ G. A. Narvaez, G. Bester, and A. Zunger, Phys. Rev. B 72, 245318 (2005).

${ }^{32}$ G. W. Bryant, N. Malkova, and J. Sims, Phys. Rev. B 88, 161301(R) (2013).

${ }^{33}$ G. Pfanner, M. Seliger, and U. Hohenester, Phys. Rev. B 78, 195410 (2008).

${ }^{34}$ F. Troiani and C. Tejedor, Phys. Rev. B 78, 155305 (2008). 